\title{
Interferon Gamma-activated Human Monocytes Downregulate Transferrin Receptors and Inhibit the Intracellular Multiplication of Legionella pneumophila by Limiting the Availability of Iron
}

Thomas F. Byrd and Marcus A. Horwitz

Division of Infectious Diseases, Department of Medicine, School of Medicine, University of California, Los Angeles, Center for the Health Sciences, Los Angeles, California 90024

\begin{abstract}
We have investigated the role of iron in the intracellular biology of Legionella pneumophila in human monocytes and in the effector arm of cell-mediated immune defense against this intracellular bacterial pathogen.

To determine if $L$. pneumophila intracellular multiplication is iron dependent, we studied the effect of the iron chelator deferoxamine on $L$. pneumophila infection of monocytes. Deferoxamine at $15 \mu \mathrm{M}$ completely inhibited $L$. pneumophila intracellular multiplication. The inhibitory effect of deferoxamine was reversed with equimolar iron-saturated transferrin but not apotransferrin.

To examine the potential role of iron in monocyte activation, we investigated the influence of iron-saturated transferrin on $L$. pneumophila multiplication in IFNr-activated monocytes. Iron transferrin, but not apotransferrin, neutralized the capacity of activated monocytes to inhibit $L$. pneumophila multiplication.

To explore a potential mechanism by which activated monocytes might limit the availability of intracellular iron, we examined transferrin receptor expression on nonactivated and activated monocytes cultured in vitro for $5 \mathrm{~d}$. By fluorescenceactivated flow cytometry, activated monocytes exhibited markedly fewer transferrin receptors than nonactivated monocytes. By Scatchard analysis of ${ }^{125} \mathrm{I}$-transferrin binding to monocytes, nonactivated monocytes had $38,300 \pm 12,700$ (mean $\pm S E$ ) transferrin binding sites, whereas activated monocytes had $10,300 \pm 1,600$, a reduction of $73 \%$. Activated and nonactivated monocytes had a similar mean $K_{d}(1.8 \pm 0.2 \mathrm{nM})$.

This study demonstrates that (a) L. pneumophila intracellular multiplication is iron dependent; (b) activated monocytes inhibit $L$. pneumophila multiplication by limiting the availability of intracellular iron; and (c) transferrin receptors are downregulated on IFN $\gamma$-activated monocytes.
\end{abstract}

This paper was presented in part at the 1987 National Meeting of the Association of American Physicians, the American Society for Clinical Investigation, and the American Federation for Clinical Research, 1-4 May 1987, Washington, DC.

Address correspondence to Dr. Thomas F. Byrd, Division of Infectious Diseases, Department of Medicine, UCLA School of Medicine, 10833 Le Conte Avenue, Los Angeles, CA 90024-1736.

Received for publication 22 September 1988 and in revised form 8 December 1988.

J. Clin. Invest.

(c) The American Society for Clinical Investigation, Inc.

0021-9738/89/05/1457/09 \$2.00

Volume 83, May 1989, 1457-1465

\section{Introduction}

Legionella pneumophila is a facultative, gram-negative, intracellular bacterial pathogen that invades the lung and parasitizes alveolar macrophages (1-3). Cell-mediated immunity appears to play a major role in host defense against $L$. pneumophila (4). Human monocytes and alveolar macrophages activated by cytokines from mitogen- or antigen-stimulated lymphocytes, or by recombinant human IFN $\gamma$, inhibit $L$. pneumophila intracellular multiplication (4-8). Although activated mononuclear phagocytes inhibit the multiplication of L. pneumophila, they do not kill the intracellular bacteria $(2,5$, 6 ). This suggests that the major contribution of cell-mediated immunity to host defense against $L$. pneumophila is to inhibit bacterial multiplication rather than kill the pathogen.

The mechanisms by which activated macrophages exert an antimicrobial effect are incompletely understood. In this paper we shall propose that activated human monocytes inhibit $L$. pneumophila intracellular multiplication by limiting the availability of iron.

L. pneumophila has a definite metabolic requirement for iron, reflected by an inability to grow on agar medium in the absence of iron supplementation (9) or in broth medium to which iron chelators, including apotransferrin, enterochelin, or deferoxamine, have been added $(10,11)$. Unlike most gram-negative bacteria, L. pneumophila does not produce siderophores, soluble iron-chelating compounds, that would allow it to remove iron in vivo from high affinity iron-binding compounds such as transferrin (11). L. pneumophila multiplication is thus restricted to environments in which iron is readily available and not bound to high affinity iron-binding compounds. The distinctive ribosome-lined phagosome in which $L$. pneumophila multiplies intracellularly is presumably one such environment in mononuclear phagocytes (12).

Mononuclear phagocytes, like other mammalian cells, acquire iron via transferrin receptors on their surface $(13,14)$. The transferrin receptor is thought to be composed of two identical polypeptide chains, each capable of binding a diferric transferrin molecule at neutral $\mathrm{pH}$ (15). The transferrin receptor-transferrin complex is endocytized, after which the endocytic vacuole is acidified. As the $\mathrm{pH}$ decreases, ferric ions dissociate from transferrin and are distributed intracellularly. The apotransferrin remains bound to the transferrin receptor until the receptor recycles to the cell surface, whereupon apotransferrin dissociates from the receptor (16). The internalized iron enters the so-called labile iron pool of the cell (17).

In this study we have explored the role of iron in the intracellular biology of $L$. pneumophila in nonactivated and IFN $\gamma$ activated human monocytes. We shall demonstrate that $(a) L$. pneumophila intracellular multiplication is iron dependent; 
(b) activated monocytes inhibit $L$. pneumophila intracellular multiplication by limiting the availability of iron; and $(c)$ transferrin receptors are markedly downregulated on activated monocytes.

\section{Methods}

Media. Egg yolk buffer with or without $1 \%$ BSA, and PBS were prepared or obtained as described (1). RPMI 1640 medium with L-glutamine (RPMI) ${ }^{1}$ (Gibco Laboratories, Grand Island, NY) was used alone, with $10 \mathrm{mM}$ Hepes buffer (Sigma Chemical Co., St. Louis, MO), or with $10 \mathrm{mM}$ Hepes buffer and $0.1 \%$ BSA (Sigma Chemical Co.). Antibiotics were not added to media in any experiment.

Tissue culture reagents. Deferoxamine mesylate (Ciba-Geigy, Summit, NJ), iron-saturated transferrin, and apotransferrin (Miles Scientific, Kanakee, IL) were dissolved in RPMI and filtered through $0.22-\mu \mathrm{m}$ filter units. Escherichia coli-derived recombinant human IFN $\gamma$, purified to homogeneity, was a gift from Genentech Inc., South San Francisco, CA. Lot D0002SF was used in initial experiments. This lot was stored at $4^{\circ} \mathrm{C}$ in PBS containing 5\% BSA (PBS-BSA), pH 6.9, at a concentration of $20 \mu \mathrm{g}$ protein $/ \mathrm{ml}$. This preparation contained 1.1 $\times 10^{4}$ antiviral units/ $\mu$ g. A second lot, K9079A, received as a lyophilized powder, was used in later experiments. It was reconstituted with sterile, "endotoxin free" water (Sigma Chemical Co.) to a concentration of $960 \mu \mathrm{g} / \mathrm{ml}$, stored at $4^{\circ} \mathrm{C}$, and diluted with PBS-BSA for use in experiments. This preparation contained $2.65 \times 10^{4}$ antiviral units $/ \mu \mathrm{g}$.

Agar. Modified charcoal yeast extract agar was prepared in 100 $\times 15-\mathrm{mm}$ bacteriologic petri dishes as described (1).

Serum. Venous blood was obtained from healthy, adult volunteers with no history of Legionnaires' disease, and serum was separated and stored at $-70^{\circ} \mathrm{C}$. In all experiments autologous serum was used.

Bacteria. L. pneumophila, Philadelphia 1 strain, serogroup 1, was grown in embryonated hens' eggs, harvested, tested for viability and for the presence of contaminating bacteria, and stored at $-70^{\circ} \mathrm{C}$ as described (1). In some experiments bacteria were partially purified by differential centrifugation just before use. In most experiments the bacteria were passed one time only on modified charcoal yeast extract agar for $80 \mathrm{~h}$, harvested, stored at $-70^{\circ}$, and thawed in a $37^{\circ} \mathrm{C}$ waterbath just before use.

Human blood mononuclear cells. Mononuclear cells used in all experiments were obtained from the blood of healthy, adult volunteers with no history of Legionnaires' disease. The blood mononuclear cell fraction was obtained by centrifugation over a Ficoll-sodium diatrizoate solution (Pharmacia Fine Chemicals, Piscataway, NJ) as previously described (1). For some experiments, monocyte- and lymphocyte-enriched populations of cells were obtained from the blood mononuclear cell fraction on preformed Percoll gradients as described (18).

Assay for iron dependence of $L$. pneumophila intracellular multiplication in monocytes. Freshly explanted monocytes were adhered to Linbro flat-bottomed wells (16 $\mathrm{mm}$ in diameter; Flow Laboratories, Inc., McLean, VA) as described (4). The monocyte monolayers containing $\sim 5 \times 10^{5}$ cells were then incubated at $37^{\circ} \mathrm{C}$ in $5 \% \mathrm{CO}_{2}-95 \%$ air in $0.5 \mathrm{ml}$ of RPMI containing $20 \%$ fresh normal human serum and either deferoxamine mesylate at a final concentration of 15,25 , or 35 $\mu \mathrm{M}$, or control medium. In some experiments iron transferrin (7.5 $\mu \mathrm{M})$ or apotransferrin $(7.5 \mu \mathrm{M})$ was added to wells containing deferoxamine. After $24 \mathrm{~h}$ L. pneumophila $\left(5 \times 10^{2} \mathrm{CFU} / \mathrm{ml}\right)$ were added to the monocytes, and the cultures were incubated at $37^{\circ} \mathrm{C}$ in $5 \%$ $\mathrm{CO}_{2}-95 \%$ air on a gyratory shaker $(100 \mathrm{rpm})$ for $1 \mathrm{~h}$ and under stationary conditions thereafter. CFU of $L$. pneumophila in each culture were determined daily as described (1). After $96 \mathrm{~h}$, the viability of monocytes was assayed by trypan blue exclusion. All experiments were run in triplicate.

1. Abbreviations used in this paper: $K_{\mathrm{a}}$, affinity constant; MPFC, mean peak fluorescent channel; RPMI, RPMI 1640 medium with L-glutamine.
Assay for the effect of iron transferrin on L. pneumophila multiplication in IFN $\gamma$-activated monocytes. Monocyte monolayers, prepared as described above, were incubated at $37^{\circ} \mathrm{C}$ in $5 \% \mathrm{CO}_{2}-95 \%$ air in RPMI containing $20 \%$ fresh normal human serum in the presence of IFN $\gamma(2 \mu \mathrm{g} / \mathrm{ml})$ or control buffer (PBS-BSA). Iron transferrin (0.07-6 $\mathrm{mg} / \mathrm{ml})$, apotransferrin $(6 \mathrm{mg} / \mathrm{ml})$, or control buffer was added to each well of the IFN $\gamma$-treated cultures. After 24 h L . pneumophila $\left(10^{4}\right.$ $\mathrm{CFU} / \mathrm{ml}$ ) were added to the cultures and CFU determined daily as above. All experiments were run in triplicate.

Assay for transferrin receptor expression on activated and nonactivated monocytes using flow cytometry. Monocyte-enriched cell suspensions were prepared as described above and cultured at a concentration of $1.5-2.0 \times 10^{6} / \mathrm{ml}$ in screw cap Teflon wells (Savillex Corp., Minnetonka, MN) in RPMI containing $20 \%$ autologous human serum in the presence of IFN $\gamma(2 \mu \mathrm{g} / \mathrm{ml})$ or control medium (PBS-BSA). The wells were loosely capped and the cells were incubated at $37^{\circ} \mathrm{C}$ in $5 \%$ $\mathrm{CO}_{2}-95 \%$ air for $5 \mathrm{~d}$. Although such cells will be referred to as monocytes in this paper, they are known to take on many of the characteristics of tissue macrophages as they mature in culture. After $5 \mathrm{~d}$ the Teflon wells were put on ice for $30 \mathrm{~min}$. The monocyte suspensions were then removed and washed twice by centrifugation at $200 \mathrm{~g}$ for 10 min at $4{ }^{\circ} \mathrm{C}$ in PBS without $\mathrm{Ca}^{2+}$ and $\mathrm{Mg}^{2+}$ ions containing $2 \% \mathrm{FCS}$ and $0.1 \%$ sodium azide. The cells were then counted, aliquoted to triplicate tubes $\left(2 \times 10^{5}\right.$ cells/tube), centrifuged at $150 \mathrm{~g}$ at $4^{\circ} \mathrm{C}$ for $5 \mathrm{~min}$, and resuspended in $100 \mu$ l PBS containing 50\% human AB serum (Irvine Scientific, Santa Ana, CA). Cells in the first tube of a triplicate set were then incubated with fluorescein-conjugated mouse anti-transferrin receptor IgG2a MAb (L01.1; Becton Dickinson Immunocytometry Systems, Mountain View, CA), cells in the second tube were incubated with fluorescein-conjugated mouse anti-limpet hemocyanin IgG2a MAb (Becton Dickinson Immunocytometry Systems) as an isotypic control, and cells in the third tube were incubated without antibody to assess autofluorescence. Cells in all three tubes were incubated in the dark for $30 \mathrm{~min}$ at $4^{\circ} \mathrm{C}$, washed twice, and resuspended in $500 \mu \mathrm{l}$ PBS. Flow cytometric analysis of the cells was then performed on a flow cytometer (Epics C; Coulter Electronics Inc., Hialeah, FL) equipped with an argon laser tuned to $488 \mathrm{~nm}$ and run at $300 \mathrm{~mW}$. A filter combination consisting of a $525 / 20-\mathrm{nm}$ band pass filter in front of the green photomultiplier tube was used. The flow cytometer was gated on forward light scatter and right angle scatter so as to exclude lymphocytes, cell debris, clumps, and nonviable cells, and the log fluorescence intensity of 5,000 monocytes from each sample was displayed as a frequency distribution histogram. Monocytes incubated without antibody and monocytes incubated with the isotypic control antibody exhibited comparably low levels of fluorescence.

Scatchard analysis of transferrin binding sites in nonactivated and activated monocytes. Monocyte-enriched cell suspensions were cultured in Teflon wells for $5 \mathrm{~d}$ with and without IFN $\gamma$ as in the previous assay. The Teflon wells were then put on ice for $30 \mathrm{~min}$ and the cell suspensions were transferred to $50-\mathrm{ml}$ polypropylene centrifuge tubes (Corning Glass Works, Corning, NY) on ice. The Teflon wells were washed twice with ice-cold RPMI and the wash was added to the centrifuge tubes. The monocytes were then washed twice with ice-cold RPMI by centrifugation at $150 \mathrm{~g}$ for $20 \mathrm{~min}$ and resuspended in icecold RPMI containing $10 \mathrm{mM}$ Hepes. Trypan blue exclusion showed that these cells were $>90 \%$ (mean, $93 \%$ ) viable; the few nonviable cells appeared to be lymphocytes. A sample from each cell suspension was removed and centrifuged onto a glass slide using a cytocentrifuge (Shandon Southern Instruments Inc., Sewickley, PA). The slides were then stained with alpha naphthyl acetate esterase (Sigma Chemical Co.), and the percentage of monocytes and lymphocytes in this preparation was determined. This percentage was used to determine the number of monocytes used in an assay; $2-5 \times 10^{5}$ monocytes were used per assay tube. Cells were then added to triplicate $17 \times 100-\mathrm{mm}$ polypropylene tubes (Falcon 2059; Becton Dickinson), brought to a volume of $500 \mu \mathrm{l}$ with RPMI-10 mM Hepes, and preincubated at $37^{\circ} \mathrm{C}$ for $90 \mathrm{~min}$ in $5 \% \mathrm{CO}_{2}-95 \%$ air. This preincubation step was performed to allow the release of previously bound transferrin from transferrin 
receptors and to allow internalized, functional transferrin receptors to recycle back to the cell surface so that these receptors would be available for binding ${ }^{125} \mathrm{I}$-transferrin in this assay. After the preincubation, 5 $\mathrm{ml}$ of RPMI- $10 \mathrm{mM}$ Hepes- $0.1 \%$ BSA at $4^{\circ} \mathrm{C}$ was immediately added to each tube, and the cells were centrifuged at $150 \mathrm{~g}$ for $10 \mathrm{~min}$. The supernatant fluid was removed and ${ }^{125} \mathrm{I}$-diferric transferrin (New England Nuclear, Boston, MA) was added to each triplicate tube. Specific activity of the preparation of ${ }^{125}$ I-transferrin used ranged from 0.5 to $0.8 \mu \mathrm{Ci} / \mu \mathrm{g}$. Unlabeled iron transferrin was added to one tube of each triplicate set at a final concentration of $10,000 \mathrm{nM}$ to control for nonspecific binding. Six sets of triplicate tubes were prepared in this way with concentrations of ${ }^{125} \mathrm{I}$-transferrin ranging from 0.1 to $6 \mathrm{nM}$. The tubes were gassed with $\mathrm{CO}_{2}$, capped, and sealed with parafilm. After a 3-h incubation period at $4^{\circ} \mathrm{C}$ the monocytes were washed twice with 4-6 ml of RPMI- $10 \mathrm{mM}$ Hepes-0.1\% BSA, suspended in $1 \mathrm{ml}$ of 1 $\mathrm{N} \mathrm{NaOH}$, transferred to $12 \times 75-\mathrm{mm}$ polystyrene tubes (Falcon 2054; Becton Dickinson), and ${ }^{125}$ I-transferrin bound was counted on a gamma counter with a counting efficiency of $68 \%$ for ${ }^{125}$ I (Beckman Biogamma II; Beckman Instruments, Inc., Fullerton, CA).

In experiments in which the binding of ${ }^{125}$ I-transferrin to nonactivated monocytes and nonactivated lymphocytes was compared, lymphocyte-enriched cell suspensions were cultured in Teflon wells as described above. After $5 \mathrm{~d}$ the lymphocytes were incubated twice, sequentially, in 35-mm plastic tissue culture dishes (Falcon 3001; Becton Dickinson) at $37^{\circ} \mathrm{C}$ for $90 \mathrm{~min}$ in $5 \% \mathrm{CO}_{2}-95 \%$ air to allow any remaining monocytes to adhere. A sample from the purified lymphocyte preparation was then centrifuged onto a glass slide with a cytocentrifuge and stained with alpha naphthyl acetate esterase stain, and the percentage of lymphocytes in this final preparation was determined. The lymphocytes were then used in the assay described above.

In some experiments the step involving culture of the monocytes in Teflon wells was eliminated. In these experiments, freshly isolated monocyte-enriched cell suspensions were added directly to $17 \times 100$ $\mathrm{mm}$ polypropylene tubes and incubated in RPMI containing $20 \%$ autologous serum in the presence or absence of IFN for $5 \mathrm{~d}$ at $37^{\circ} \mathrm{C}$ in $5 \%$ $\mathrm{CO}_{2}-95 \%$ air. The monocytes were then washed twice to remove serum, resuspended in $500 \mu \mathrm{l}$ of RPMI- $10 \mathrm{mM}$ Hepes, and preincubated at $37^{\circ} \mathrm{C}$ for $90 \mathrm{~min}$ in $5 \% \mathrm{CO}_{2}-95 \%$ air. The remaining part of the assay was the same as described above.

\section{Results}

L. pneumophila intracellular multiplication is iron dependent. To determine if $L$. pneumophila requires access to intracellular iron to multiply, we examined the influence of the iron chelator deferoxamine on L. pneumophila intracellular multiplication. Deferoxamine chelates iron in what has been characterized as the labile iron pool $(17,19-21)$. We infected monocytes with $L$. pneumophila in the presence or absence of deferoxamine and assayed intracellular multiplication (Fig. 1).

L. pneumophila multiplied several logs in control cultures, but multiplication was completely inhibited in cultures treated with $15 \mu \mathrm{M}$ deferoxamine. The effect of deferoxamine was not due to death of the monocytes, since these cells remained viable throughout the course of the experiment, as assayed by trypan blue exclusion. Moreover, whereas control monolayers were destroyed by multiplying L. pneumophila, deferoxamine-treated monolayers remained intact and morphologically normal.

Higher concentrations of deferoxamine $(20,25$, and 35 $\mu \mathrm{M})$ produced similar inhibition of $L$. pneumophila multiplication. The 20- and $25-\mu \mathrm{M}$ concentrations of deferoxamine had no apparent toxic effects on monocyte monolayers and did not decrease monocyte viability by trypan blue exlusion. However, the 35- $\mu \mathrm{M}$ concentration of deferoxamine caused

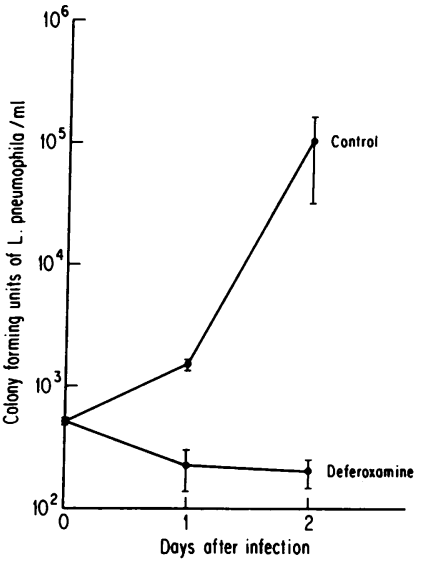

Figure 1. Deferoxamine inhibits the intracellular multiplication of $L$. pneumophila in human monocytes. Monocytes in monolayer culture were incubated in the presence or absence (control) of $15 \mu \mathrm{M}$ deferoxamine. After $24 \mathrm{~h}$ the monocytes were infected with L. pneumophila as described in the text. CFU of L. pneumophila were determined daily. Data are the mean \pm SEM for triplicate cultures.

some loss of adherent cells from the monolayers and aggregation of the remaining viable cells.

To confirm that the effect of deferoxamine resulted from its capacity to chelate iron, we added $7.5 \mu \mathrm{M}$ iron-saturated transferrin (equimolar for iron-binding capacity with deferoxamine) to monocyte cultures treated with $15 \mu \mathrm{M}$ deferoxamine (Fig. 2). Under physiologic conditions, deferoxamine does not remove appreciable amounts of iron from transferrin (22). We added apotransferrin (99\% unsaturated) to control cultures.

Iron transferrin completely reversed the capacity of deferoxamine to inhibit L. pneumophila multiplication (Fig. 2). In the presence of both deferoxamine and iron-saturated transferrin, L. pneumophila multiplied to the same extent as in untreated control cultures. In contrast, apotransferrin (7.5 $\mu \mathrm{M})$ had only a minimal effect on deferoxamine-treated monocytes.

Thus, L. pneumophila multiplication in monocytes is dependent upon the availability of intracellular iron.

The capacity of activated monocytes to inhibit L. pneumophila multiplication is reversed by iron transferrin. We then hypothesized that iron may play an important role in monocyte activation against $L$. pneumophila. To explore this, we studied the influence of iron transferrin on L. pneumophila multiplication in IFN $\gamma$-activated monocytes (Fig. 3).

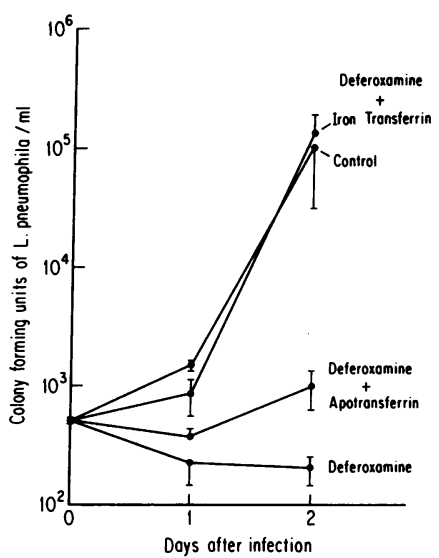

Figure 2. Deferoxamine inhibition of $L$. pneumophila multiplication in human monocytes is reversed by iron-saturated transferrin. Monocytes in monolayer culture were incubated in medium alone (control), or in medium containing $15 \mu \mathrm{M}$ deferoxamine, $15 \mu \mathrm{M}$ deferoxamine and $7.5 \mu \mathrm{M}$ iron transferrin, or $15 \mu \mathrm{M}$ deferoxamine and $7.5 \mu \mathrm{M}$ apotransferrin. After $24 \mathrm{~h}$ the monocytes were infected with $L$. pneumophila as described in the text. CFU of $L$. pneumo phila were determined daily. Data are mean \pm SEM for triplicate cultures. 


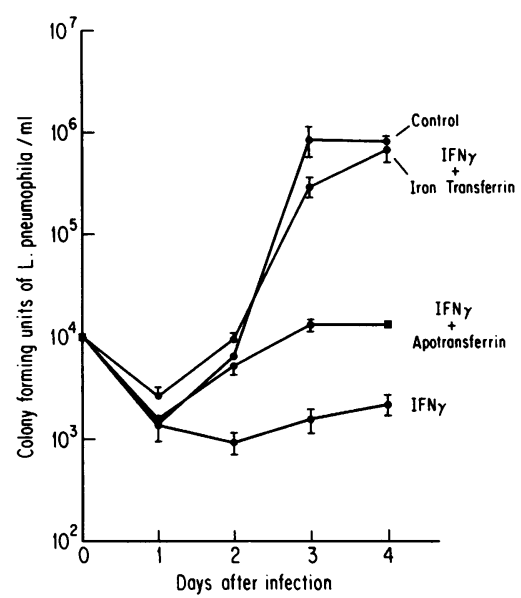

Figure 3. The capacity of IFN $\gamma$-activated human monocytes to inhibit $L$. pneumophila intracellular multiplication is reversed by ironsaturated transferrin. Monocytes in monolayer culture were incubated in medium alone (control) or activated by incubation in medium containing human recombinant IFN $\gamma$. At the same time, IFN $\gamma$ treated monocytes were incubated with control medium, iron-saturated transferrin $(6 \mathrm{mg} / \mathrm{ml})$, or apotransferrin $(6 \mathrm{mg} / \mathrm{ml})$. After $24 \mathrm{~h}$ all cultures were infected with $L$. pneumophila. CFU of $L$. pneumophila were determined daily. Data are mean \pm SEM for triplicate cultures.

Iron transferrin at a concentration of $6 \mathrm{mg} / \mathrm{ml}$, approximately twice the concentration in normal human serum, completely reversed the capacity of IFN $\gamma$-activated monocytes to inhibit $L$. pneumophila multiplication. In contrast, apotransferrin at a concentration of $6 \mathrm{mg} / \mathrm{ml}$ had only a minimal effect on L. pneumophila multiplication in activated monocytes.

The neutralizing effect of iron transferrin on monocyte activation was dose dependent (Fig. 4). At a concentration of 6 $\mathrm{mg} / \mathrm{ml}$, iron transferrin completely reversed the capacity of IFN $\gamma$-activated monocytes to inhibit $L$. pneumophila multiplication. At concentrations of 2.0 and $0.7 \mathrm{mg} / \mathrm{ml}$, iron transferrin partially reversed monocyte activation. At concentrations of $0.2 \mathrm{mg} / \mathrm{ml}$ or less, iron transferrin had little or no effect on the capacity of activated monocytes to inhibit L. pneumophila multiplication.

These experiments indicated that IFN $\gamma$-activated monocytes inhibit $L$. pneumophila intracellular multiplication by limiting the availability of intracellular iron.

Transferrin receptors are downregulated on IFN $\gamma$-activated monocytes. We hypothesized that one way that activated monocytes might limit the availability of intracellular iron is

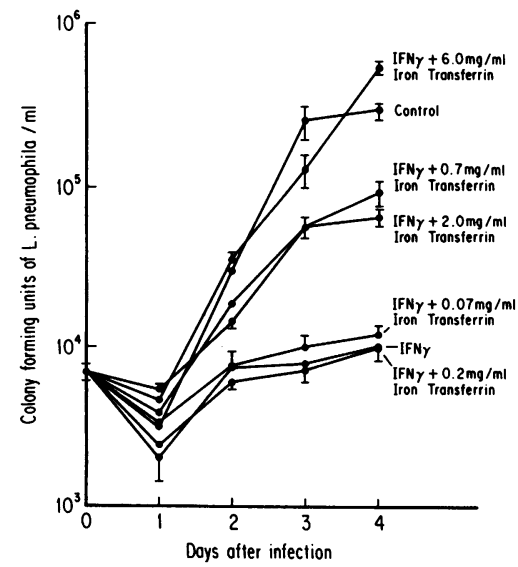

Figure 4. Dose-dependent reversal of IFN $\gamma$ mediated inhibition of L. pneumophila multiplication in human monocytes by iron-saturated transferrin. Monocytes were incubated in medium alone (control) or activated by incubation with IFN $\gamma$ as in the previous figure. At the same time, the IFN $\gamma$-treated monocytes were incubated with various concentrations of iron-saturated

transferrin ranging from 0.07 to $6.0 \mathrm{mg} / \mathrm{ml}$. After $24 \mathrm{~h}$ all cultures were infected with $L$. pneumophila. CFU of $L$. pneumophila was determined daily. Data are the mean \pm SEM for triplicate cultures. by downregulating transferrin receptors on their surface. To examine this possibility, we investigated transferrin receptor expression on activated and nonactivated monocytes by fluorescence-activated flow cytometry.

In two experiments on one subject (subject 3 in Table I, see below) we generated histograms of monocytes treated with anti-transferrin receptor MAb and control monocytes treated with an isotypic control MAb and determined the shift in mean peak fluorescent channel (MPFC) between the two histograms. In both experiments activated monocytes exhibited a markedly decreased shift in MPFC consistent with decreased transferrin receptor expression (Fig. 5). Whereas nonactivated monocytes exhibited shifts in MPFC of 36 and 26 in the two experiments, activated monocytes exhibited shifts of 18 and 14 , respectively.

In these experiments we also determined the percentage of monocytes treated with anti-transferrin receptor MAb that fluoresced with an intensity $>98 \%$ of monocytes treated with the isotypic control MAb. Whereas 19 and $22 \%$ of nonactivated monocytes fluoresced above this level of intensity in the two experiments, only 5 and $8 \%$, respectively, of activated monocytes did so.

Thus, activated monocytes exhibit decreased transferrin receptor expression.

Transferrin receptors on activated monocytes have normal affinity for transferrin but are reduced in number by Scatchard analysis. To quantitate the reduction in transferrin receptor expression more precisely and to assess the binding affinities of transferrin receptors on activated and nonactivated monocytes, we examined transferrin receptors by Scatchard analysis (23). We first incubated monocytes with a constant amount of ${ }^{125}$ I-transferrin at $4^{\circ} \mathrm{C}$ (Fig. 6). Both activated and nonacti-

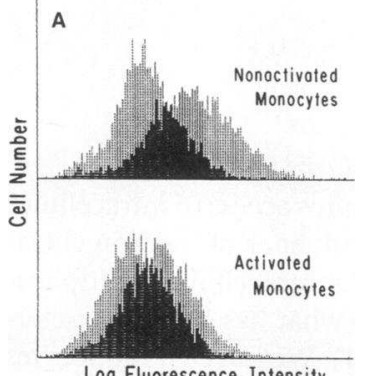

Log Fluorescence Intensity

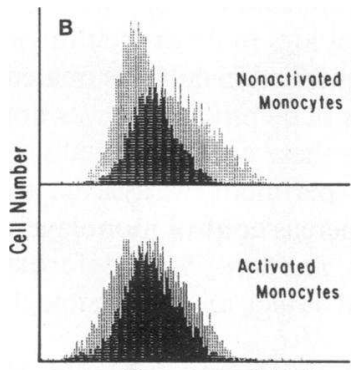

Log Fluorescence Intensity
Figure 5. Transferrin receptor expression is downregulated on IFN $\gamma$-activated human monocytes. In two independent experiments $(A$ and $B)$ monocytes from the same individual were incubated for 5 $d$ in Teflon wells in control medium (nonactivated monocytes) or in medium containing IFN $\gamma$ (activated monocytes). The activated and nonactivated monocytes were then incubated with fluoresceinconjugated anti-transferrin receptor MAb or with a fluorescein-conjugated isotypic control MAb and analyzed by fluorescence-activated flow cytometry. The figures depict fluorescent histograms of nonactivated monocytes (upper curves) and activated monocytes (lower curves). In each of the four sets of histograms, the left curve represents the fluorescence intensity of monocytes incubated with the isotypic control antibody and the right curve represents the fluorescence intensity of monocytes treated with anti-transferrin receptor antibody. To the right of each set of histograms, the shift in mean peak fluorescent channel is shown. 


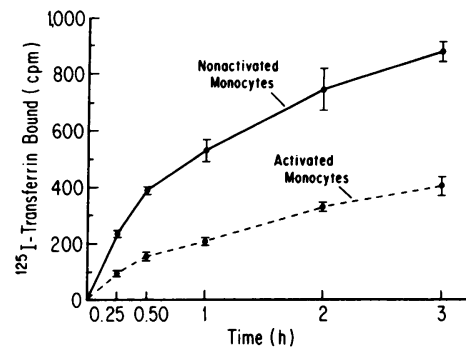

Figure 6. Time course of ${ }^{125}$ I-transferrin binding to activated and nonactivated monocytes. Nonactivated monocytes and monocytes activated with IFN $\gamma$ for $5 \mathrm{~d}$ were prepared as described in the text. Then, $3 \times 10^{5}$ monocytes in $500 \mu \mathrm{l}$ of culture medium were incubated with $3 \mathrm{nM}^{125}$ I-trans-

ferrin $\left(10^{5} \mathrm{cpm} /\right.$ tube $)$ at $4^{\circ} \mathrm{C}$ for $0.25-3 \mathrm{~h}$ as indicated. The amount of ${ }^{125} \mathrm{I}$-transferrin bound to monocytes was measured at each time point. Nonspecific binding was assessed by incubating monocytes with ${ }^{125}$ I-transferrin in replicate tubes in the presence of $10,000 \mathrm{nM}$ unlabeled transferrin. Data are the mean \pm SEM for duplicate measurements corrected for nonspecific binding and background counts.

vated monocytes bound increasing amounts of ${ }^{125} \mathrm{I}$-transferrin with time; binding was near maximal by $3 \mathrm{~h}$. Nonactivated monocytes bound more ${ }^{125}$ I-transferrin than activated monocytes.

To assess specificity of ${ }^{125}$ I-transferrin binding, we incubated monocytes with a constant amount of ${ }^{125} \mathrm{I}$-transferrin and increasing amounts of unlabeled transferrin (Fig. 7). For both activated and nonactivated monocytes, the amount of cell-bound ${ }^{125} \mathrm{I}$-transferrin decreased with increasing concentration of unlabeled transferrin, indicating that the binding of transferrin to the monocytes was specific.

To assess receptor number and affinity by Scatchard analysis, we incubated activated and nonactivated monocytes with various concentrations of ${ }^{125} \mathrm{I}$-transferrin for $3 \mathrm{~h}$ and measured the amount of transferrin bound to the cells (Fig. 8). Preliminary experiments showed that specific binding to activated and nonactivated monocytes reached a plateau at $6 \mathrm{nM}$ transferrin (i.e., transferrin binding was the same at 6 and $12 \mathrm{nM}$ transferrin, indicating that the transferrin receptors were saturable). Therefore, in subsequent experiments, including the one shown in Fig. 8, binding was measured at concentrations of transferrin up to $6 \mathrm{nM}$. Scatchard analysis of this equilibrium binding data revealed a single class of high affinity receptors on both activated and nonactivated monocytes (Fig. 9). The receptors on both types of monocytes had a similar $K_{\mathrm{d}}$, but activated monocytes had fewer receptors.

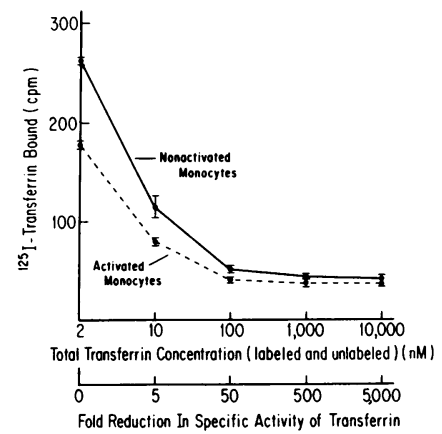

Figure 7. Specificity of ${ }^{125}$ Itransferrin binding to activated and nonactivated monocytes. Nonactivated and activated monocytes $\left(4 \times 10^{5}\right.$ in $500 \mu$ of medium), prepared as in the previous figure, were incubated at $4^{\circ} \mathrm{C}$ for $3 \mathrm{~h}$ with 2 $\mathrm{nM}^{125}$ I-transferrin $\left(10^{4} \mathrm{cpm} /\right.$ tube) and various amounts of unlabeled transferrin such that specific activity of labeled transferrin was reduced from

5- to 5,000-fold as indicated. For each concentration of transferrin, the amount of ${ }^{125}$ I-transferrin bound to monocytes was measured. Data are the mean \pm SEM for duplicate determinations.

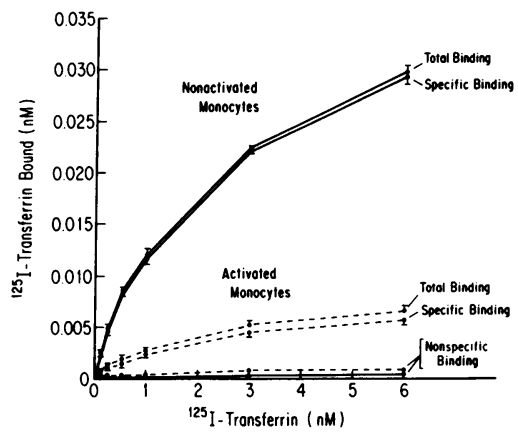

Figure 8. Equilibrium binding of ${ }^{125}$ I-transferrin to activated and nonactivated monocytes. Activated and nonactivated monocytes $\left(2 \times 10^{5}\right.$ in $\left.500 \mu\right)$ of medium) were incubated at $4^{\circ}$ for $3 \mathrm{~h}$ with various concentrations of ${ }^{125}$ I-transferrin (specific activity, 0.6

$\mu \mathrm{Ci} / \mu \mathrm{g})$ as indicated.

The total amount of ${ }^{125}$ I-transferrin bound to monocytes was measured at each concentration of ${ }^{125}$ I-transferrin. Nonspecific binding was assessed by incubating monocytes with ${ }^{125} \mathrm{I}$-transferrin in replicate tubes in the presence of $10,000 \mathrm{nM}$ unlabeled transferrin. Specific binding was obtained by subtracting nonspecific binding from total binding. Data are the mean \pm SEM for duplicate measurements corrected for background counts.

To describe transferrin receptor number and affinity on activated and nonactivated human monocytes more fully, we performed Scatchard analysis twice on 5-d monocytes from each of four subjects in eight independent experiments (Table I). In any one subject there was little variation in transferrin receptor number per monocyte from one experiment to the next. However, there was wide variation in the number of receptors among different subjects. The mean number of transferrin binding sites on nonactivated monocytes was $38,300 \pm 12,700$ (mean \pm SEM) with a range of $13,100-69,000$. Whatever the number of transferrin receptors on nonactivated monocytes, the number of receptors on activated monocytes was markedly decreased in all subjects. The mean number of transferrin binding sites on activated monocytes was $10,300 \pm 1,600$, with a range of $5,600-12,300$. This represented a mean decrease of $73 \%$. (Assuming two transferrin binding sites per receptor, the number of receptors on activated and nonactivated monocytes would be half the values stated above.)

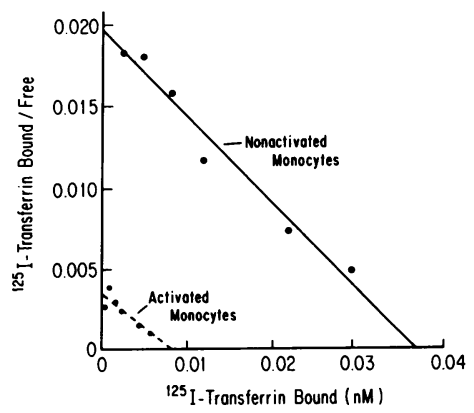

Figure 9. Scatchard plot of equilibrium binding of transferrin to activated and nonactivated monocytes. Data from Fig. 8 were used to plot the ratio of bound transferrin to free transferrin against the amount of bound transferrin at each concentration of ${ }^{125}$ I-transferrin. Linear regression was used to find the line of best fit. The correlation coefficient $(r)$ was 0.982 for nonactivated monocytes and 0.873 for activated monocytes. The $K_{\mathrm{d}}$ (inverse of the slope) was $1.87 \mathrm{nM}$ for nonactivated monocytes and $2.34 \mathrm{nM}$ for activated monocytes. The number of transferrin binding sites (calculated from the $x$ intercept) was 55,200 for nonactivated monocytes and 12,200 for activated monocytes. The experiment is representative of 11 independent measurements of transferrin binding sites and $K_{\mathrm{d}}$ for human monocytes. 
Table I. Transferrin Receptor Number Is Decreased on IFN $\gamma$-activated Monocytes

\begin{tabular}{|c|c|c|c|c|}
\hline Subject & $\begin{array}{c}\text { Status of } \\
\text { monocytes }\end{array}$ & $\begin{array}{c}\text { Number of } \\
\text { transferrin binding } \\
\text { sites per monocyte } \\
\text { (mean } \pm \text { SD) }\end{array}$ & $\begin{array}{c}K_{\mathrm{d}} \\
(\mathrm{Mean} \pm \mathrm{SD})\end{array}$ & $\begin{array}{l}\text { Percent decrease } \\
\text { in transferrin } \\
\text { binding sites } \\
\text { per monocyte } \\
\text { with activation }\end{array}$ \\
\hline & & \multicolumn{3}{|c|}{$n M$} \\
\hline \multirow[t]{2}{*}{1} & Nonactivated & $69,000 \pm 4,800$ & $1.92 \pm 1.03$ & $82 \%$ \\
\hline & Activated & $12,300 \pm 100$ & $2.16 \pm 0.97$ & \\
\hline \multirow[t]{2}{*}{2} & Nonactivated & $48,500 \pm 9,600$ & $2.39 \pm 0.73$ & $75 \%$ \\
\hline & Activated & $11,900 \pm 600$ & $2.28 \pm 0.08$ & \\
\hline \multirow[t]{2}{*}{3} & Nonactivated & $22,600 \pm 800$ & $1.81 \pm 0.35$ & $49 \%$ \\
\hline & Activated & $11,600 \pm 800$ & $1.54 \pm 0.10$ & \\
\hline \multirow[t]{2}{*}{4} & Nonactivated & $13,100 \pm 3,600$ & $1.23 \pm 0.67$ & $57 \%$ \\
\hline & Activated & $5,600 \pm 4,200$ & $1.38 \pm 1.32$ & \\
\hline \multirow[t]{2}{*}{ Total } & Nonactivated & $38,300 \pm 12,700$ & $1.84 \pm 0.24$ & $73 \%$ \\
\hline & Activated & $10,300 \pm 1,600$ & $1.84 \pm 0.22$ & \\
\hline
\end{tabular}

Transferrin binding sites on nonactivated and IFN $\gamma$-activated monocytes were measured twice on each of four subjects in eight independent experiments by Scatchard analysis of ${ }^{125}$ I-transferrin equilibrium binding. Data are the mean and SD of the two measurements of transferrin binding sites and $K_{d}$ for each subject, and the mean and SEM of transferrin binding sites and $K_{d}$ for all four subjects.

The $K_{d}$ of transferrin receptors of monocytes showed relatively little variation among subjects. The mean $K_{d}$ was $1.84 \pm 0.24 \mathrm{nM}$ for nonactivated monocytes and was not significantly different for activated monocytes.

To assess the potential influence of transferrin receptors from contaminating lymphocytes on the results of this assay, we performed Scatchard analysis on a purified population (99.9\%) of 5-d-old lymphocytes and on 5-d-old monocytes from the same individual at the same time. The lymphocytes had $<400$ transferrin binding sites per cell, compared with 21,600 per monocyte. Thus, the contribution of transferrin receptors from contaminating lymphocytes was minimal in this assay.

Since monocytes cultured in Teflon wells for $5 \mathrm{~d}$ adhere to some extent, we wanted to insure that our results showing transferrin receptor downregulation on IFN $\gamma$-activated monocytes relative to nonactivated monocytes were representative of the total monocyte population cultured, and not influenced by selection of an unrepresentative, less adherent monocyte population. To study a wholly unselected population of monocytes, we eliminated the step that required harvesting adherent cells from Teflon wells. Instead, we added monocyte-enriched suspensions directly to the assay tubes and incubated them for $5 \mathrm{~d}$ in these tubes before performing the assay. We performed this assay on subjects 2,3 , and 4 . Scatchard analysis revealed similar relative numbers of transferrin receptors on monocytes of the three subjects, as was observed with the initial assay (subject $2>$ subject $3>$ subject 4 ). The percent decrease in transferrin receptor number with IFN activation was also similar to that observed in the initial assay. Subject 2 had a $79 \%$ decrease in transferrin receptor number on activated monocytes, subject 3 had a $49 \%$ decrease, and subject 4 had a $38 \%$ decrease. Thus, the monocytes cultured in Teflon wells and then examined in the initial assay were representative of the originally isolated monocyte population.

\section{Discussion}

Our study demonstrates that L. pneumophila intracellular multiplication is iron dependent. Deferoxamine inhibits $L$. pneumophila intracellular multiplication and iron transferrin reverses this inhibition. Deferoxamine chelates iron in the socalled labile iron pool. This pool consists of iron that is immediately available to the cell for metabolic processes (17). Such iron is in a readily transportable form rather than in storage compounds such as ferritin or hemosiderin (17, 19-21). Iron released from endocytized iron transferrin immediately enters this pool before it is used for metabolic processes or bound to ferritin (17). Deferoxamine does not chelate a significant amount of iron bound to transferrin under physiologic conditions (22), nor does it evidently remove iron directly from ferritin (17). That deferoxamine inhibits L. pneumophila multiplication therefore indicates that $L$. pneumophila derives its iron from the labile iron pool.

After phagocytosis, L. pneumophila resides in a phagosome that interacts sequentially with monocyte smooth vesicles, mitochondria, and ribosomes, a process culminating in the formation of a ribosome-lined replicative phagosome (12). This phagosome does not fuse with lysosomes (24), and it has a pH of $\sim 6.1$ (25). After endocytosis, transferrin segregates to a juxtanuclear, para-Golgi compartment $(26,27)$ with approximately the same $\mathrm{pH}$ as the $L$. pneumophila phagosome (27). It is possible that the L. pneumophila phagosome is in contiguity with the intracellular compartment through which iron transferrin passes. If so, then L. pneumophila would have access to iron as it dissociates from transferrin. Alternatively, L. pneumophila may acquire iron at some point subsequent to the release of iron from transferrin. The immediate fate of iron that dissociates from transferrin is not known. It is presumably transported to its various destinations in the cell by an as yet uncharacterized intracellular shuttle system.

L. pneumophila multiplies exclusively intracellularly under tissue culture conditions, and probably also in nature. Our study indicates that, intracellularly, L. pneumophila has access to the labile iron pool. The bacterium may thus have no need for siderophores to remove iron from iron transferrin or other iron-binding compounds in the extracellular environment. Thus, it is perhaps not surprising that L. pneumophila does not produce siderophores or possess receptors for any of the known types of iron-chelating siderophores that promote extracellular iron acquisition by bacteria (11).

Deferoxamine has been shown to inhibit the intracellular multiplication of other parasites including Trypanosoma cruzi, which, like L. pneumophila, is an intracellular parasite of mononuclear phagocytes. Deferoxamine inhibited the multiplication of $T$. cruzi amastigotes without inducing toxic effects in mouse peritoneal macrophages at concentrations of 17 and $33 \mu \mathrm{M}$, a range similar to that used in our study (28). Deferoxamine has also been found to inhibit the multiplication of Plasmodium falciparum in human erythrocytes at concentrations as low as $15 \mu \mathrm{M}$ (29). Interestingly, in studies of the effect of deferoxamine on Plasmodium berghei infection in rats, the size of the labile iron pool in the reticulocyte has been found to be relatively stable irrespective of host iron status (21). This suggests that host iron status may be relatively un- 
important in infection of reticulocytes by the malaria parasite and, by analogy, in infection of mononuclear phagocytes by other intracellular pathogens such as $L$. pneumophila. Thus, exogenous factors such as deferoxamine, or endogenous factors such as IFN $\gamma$, which act to modify the size of the labile iron pool or the availability of intracellular iron, may be a more important influence on the course of intracellular infection than total body iron stores.

Our study demonstrates that iron transferrin, at a concentration approximately twice that in serum, completely reverses the capacity of IFN $\gamma$-activated monocytes to inhibit L. pneumophila multiplication. Apotransferrin is unable to reverse the inhibitory effect of activation. The most likely explanation for the effect of iron transferrin is that it overcomes a limitation in intracellular iron availability imposed on L. pneumophila by the activated monocyte. The effect of iron transferrin is probably not due to a toxic effect on monocytes for several reasons. First, monocytes treated with iron transferrin were viable and did not exhibit morphologic signs of toxicity. Second, the reversal of activation by transferrin was reflected by multiplication of $L$. pneumophila, which requires live, metabolically intact monocytes to replicate (1). Third, lower concentrations of iron transferrin, 0.7 and $2.0 \mathrm{mg} / \mathrm{ml}$, in the physiologic range, partially reversed the inhibitory effect of activation.

The effect of iron transferrin is also probably not due to impairment of oxidative killing mechanisms of the activated macrophage for several reasons. First, iron plays a role in the generation of toxic oxidative metabolites associated with the respiratory burst. Specifically, in the Fenton reaction, ferric iron reacts with hydrogen peroxide to produce hydroxyl radical $(30,31)$. In vitro, under cell-free conditions, hydroxyl radical is one of the most active oxidative metabolites against $L$. pneumophila (32). Were iron derived from iron transferrin influencing this system in monocytes, it should if anything reduce $L$. pneumophila viability from increased generation of hydroxyl radical. Instead, iron transferrin promotes $L$. pneumophila multiplication. Second, oxidative metabolites exert a cidal effect on microorganisms. This is inconsistent with the antimicrobial effect of activated mononuclear phagocytes on L. pneumophila. Activated monocytes and alveolar macrophages inhibit $L$. pneumophila multiplication but do not kill the bacteria $(2,5,6)$. Third, it is questionable whether the respiratory burst plays a significant role in host defense against L. pneumophila. Phagocytosis of L. pneumophila is mediated by complement receptors (CR1 and CR3) on the phagocyte and complement component $\mathrm{C} 3$ on the bacterium (33). Ligation of complement receptors by complement does not result in the release of oxidative metabolites, including hydrogen peroxide and superoxide, or mediators of inflammation such as AA metabolites (34-36). Thus, organisms entering phagocytes by this pathway may avoid the toxic consequences of the respiratory burst. Consistent with this, broth-grown $L$. pneumophila trigger a markedly reduced oxidative burst in primate alveolar macrophages (37).

On the basis of these studies, we propose that activated monocytes inhibit L. pneumophila intracellular multiplication by limiting the availability of iron. This proposed new mechanism of macrophage activation may have broad applicability to intracellular parasites, depending perhaps on the magnitude of their intracellular iron requirement and their location within the mononuclear phagocyte. Intracellular parasites occupy at least three distinctive compartments in the mononu- clear phagocyte. L. pneumophila, Toxoplasma gondii, Chlamydia psittaci, and Mycobacterium tuberculosis multiply within a phagosome that does not fuse with lysosomes $(24$, $38-40)$; leishmania species reside in a phagolysosome $(41,42)$; and $T$. cruzi exit the phagosome to multiply free in the cytoplasm $(43,44)$. It is possible that iron availability differs in these various compartments.

Two other potential mechanisms by which activated mononuclear phagocytes exert an antimicrobial effect against intracellular pathogens have been reported. First, oxidative metabolites have been implicated in the killing of $T$. cruzi on the basis of studies demonstrating that the capacity of activated mouse peritoneal macrophages to release hydrogen peroxide in response to phorbol myristate acetate is correlated with their capacity to kill this parasite (45). As noted above, this potential antimicrobial mechanism seems applicable to situations where intracellular parasites are killed but not to situations in which parasites are only inhibited from multiplying. Second, tryptophan deprivation has been investigated as an antimicrobial mechanism of IFN $\gamma$-activated cells. IFN $\gamma$ induces an enzyme, indoleamine 2,3-dioxygenase, in human fibroblasts (46), a human uroepithelial cell line (47), and human PBMC (48), that catalyzes the breakdown of tryptophan. Consequently, IFN $\gamma$-activated fibroblasts and uroepithelial cells are thought to inhibit the multiplication of $T$. gondii and $C$. psittaci, respectively, by nutritionally depriving these organisms of tryptophan $(46,47)$. However, the role of tryptophan deprivation in the microbial activity of human mononuclear phagocytes is questionable. In mononuclear phagocytes, IFN alpha and beta induce indoleamine 2,3-dioxygenase activity comparable to that of IFN $\gamma$, but unlike IFN $\gamma$ these IFNs do not induce appreciable antitoxoplasma activity $(49,50)$. Moreover, the addition of tryptophan to the media of IFN $\gamma$-activated monocytes does not reverse their antitoxoplasma activity $(50,51)$; nor does actinomycin D-induced blockade of tryptophan degradation (51). Thus, tryptophan degradation may not be in the repertoire of mechanisms by which activated mononuclear phagocytes exert an antimicrobial effect.

As a first step toward understanding the mechanism by which activated monocytes limit iron availability, we studied the effect of activation on transferrin receptor expression. Both human monocytes and alveolar macrophages, the host cells for L. pneumophila in the lung, express transferrin receptors. In a study by Hirata et al., human alveolar macrophages obtained by bronchoalveolar lavage expressed $64,000-112,000$ transferrin binding sites/cell (32,000-56,000 transferrin receptors/cell) with an affinity constant $\left(K_{\mathrm{a}}\right)$ of $4.5 \times 10^{8} \mathrm{M}^{-1}(13)$. In nonactivated human monocytes, transferrin receptor expression increases with maturation. In the study by Hirata et al., freshly explanted human monocytes expressed no detectable transferrin receptors, whereas monocytes from one subject cultured in vitro for $14 \mathrm{~d}$ expressed 72,000 transferrin binding sites/cell (36,000 transferrin receptors/cell) with a $K_{\mathrm{a}}$ of $6.7 \times 10^{8} \mathrm{M}^{-1}$. In our study, nonactivated monocytes cultured in vitro for $5 \mathrm{~d}$ expressed a mean of 38,300 transferrin binding sites/cell with a mean $K_{\mathrm{d}}$ of $1.84 \mathrm{nM}\left(K_{\mathrm{a}}\right.$ of $\left.5.43 \times 10^{8} \mathrm{M}^{-1}\right)$.

We observed considerable individual variation in the number of transferrin receptors per cell on nonactivated monocytes. Possibly, monocytes expressing low numbers of transferrin receptors are nonpermissive to $L$. pneumophila intracellular multiplication. Although one case constitutes little 
evidence for such a hypothesis, it is nevertheless interesting that subject 4's monocytes, which had the lowest number of transferrin receptors, are the only ones we have encountered that are nonpermissive for L. pneumophila multiplication.

Our study demonstrates that activated monocytes markedly downregulate the number of transferrin receptors on their surface. The mean number of transferrin binding sites on activated monocytes was reduced by $73 \%$. This suggests that one mechanism by which activated monocytes decrease the availability of intracellular iron is by modulation of the receptor that mediates endocytosis of iron.

Our finding that transferrin receptor expression is downregulated on activated human monocytes is consistent with the results of studies by Hamilton et al. on activated mouse peritoneal macrophages. These investigators showed that mouse peritoneal macrophages elicited with BCG or heat-killed Propionibacterium acnes, or activated directly with recombinant mouse IFN $\gamma$, have markedly fewer transferrin receptors than mouse peritoneal macrophages elicited with thioglycollate $(52,53)$.

In addition to downregulating transferrin receptors, activated monocytes may use other mechanisms to decrease the availability of iron to intracellular L. pneumophila. For example, activated monocytes might alter the endocytic pathway through which transferrin passes, diverting it away from the compartment in which L. pneumophila resides. Also, activated monocytes might decrease the size of the labile iron pool by altering the production of iron storage proteins such as ferritin. Further studies are underway to evaluate the role of these other potential mechanisms by which activated monocytes may limit the availability of iron.

In conclusion, our study strongly supports the hypothesis that activated monocytes inhibit L. pneumophila intracellular multiplication by limiting the availability of intracellular iron. It seems likely that activated mononuclear phagocytes possess a repertoire of antimicrobial mechanisms. The one most effective against a particular pathogen may reflect the pathogen's individual nutritional and physiologic requirements and the pathogen's complement of innate defenses against oxidative metabolites (e.g., catalase, superoxide dismutase, glutathione peroxidase, glutathione reductase, and glutathione) and nonoxidative antimicrobial substances.

\section{Acknowledgments}

We are grateful to Ms. Barbara Jane Dillon and Ms. Debora Gloria for expert technical assistance with experiments and to Ms. Ingrid Schimidt for assistance with flow cytometry.

This work was supported by grant AI-22421 from the National Institutes of Health. Dr. Horwitz is Gordon MacDonald Scholar at University of California, Los Angeles, and recipient of a Faculty Research Award from the American Cancer Society.

\section{References}

1. Horwitz, M. A., and S. C. Silverstein. 1980. The Legionnaires' disease bacterium (Legionella pneumophila) multiplies intracellularly in human monocytes. J. Clin. Invest. 66:441-450.

2. Nash, T. W., D. M. Libby, and M. A. Horwitz. 1984. Interaction between the Legionnaires' disease bacterium (Legionella pneumophila) and human alveolar macrophages. Influence of antibody, lymphokines and hydrocortisone. J. Clin. Invest. 74:771-782.
3. Winn, W. C., and R. L. Myerowitz. 1981. The pathology of Legionella pneumonias. Hum. Pathol. 12:401-409.

4. Horwitz, M. A. 1983. Cell-mediated immunity in Legionnaires' disease. J. Clin. Invest. 71:1686-1696.

5. Horwitz, M. A., and S. C. Silverstein. 1981. Activated human monocytes inhibit the intracellular multiplication of the Legionnaires' disease bacterium. J. Exp. Med. 154:1618-1635.

6. Bhardwaj, N., T. W. Nash, and M. A. Horwitz. 1986. Interferon-gamma-activated human monocytes inhibit the intracellular multiplication of Legionella pneumophila. J. Immunol. 137:26622669.

7. Jensen, W. A., R. M. Rose, A. S. Wasserman, T. H. Kalb, K. Anton, and H. G. Remold. 1987. In vitro activation of the antibacterial activity of human pulmonary macrophages by recombinant gamma interferon. J. Infect. Dis. 155:574-577.

8. Nash, T. W., D. M. Libby, and M. A. Horwitz. 1988. IFNgamma activated human alveolar macrophages inhibit the intracellular multiplication of Legionella pneumophila. J. Immunol. 140:39783981.

9. Feeley, J. C., G. W. Gorman, R. E. Weaver, D. C. Mackel, and H. W. Smith. 1978. Primary isolation media for Legionnaires' disease bacterium. J. Clin. Microbiol. 8:320-325.

10. Quinn, F. D., and E. D. Weinberg. 1988. Killing of Legionella pneumophila by human serum and iron binding agents. Curr. Microbiol. 17:111-116.

11. Reeves, M. W., L. P. Pine, J. B. Neilands, and A. Balows. 1983. Absence of siderophore activity in Legionella species grown in iron deficient media. J. Bacteriol. 154:324-329.

12. Horwitz, M. A. 1983. Formation of a novel phagosome by the Legionnaires' disease bacterium (Legionella pneumophila) in human monocytes. J. Exp. Med. 158:1319-1331.

13. Hirata, T., P. B. Bitterman, J. Mornex, and R. G. Crystal. 1986. Expression of the transferrin receptor gene during the process of mononuclear phagocyte maturation. J. Immunol. 136:1339-1345.

14. Andreesen, R., H. Osterholz, K. J. Bodemann, K. J. Bross, U. Costabel, and G. W. Lohr. 1984. Expression of transferrin receptors and intracellular ferritin during terminal differentiation of human monocytes. Blut. 49:195-202.

15. Newman, R., C. Schneider, R. Sutherland, L. Vodinelich, and M. Greaves. 1982. The transferrin receptor. Trends Biochem. Sci. 7:397-400.

16. Dautry-Varsat, A., A. Ciechanover, and H. F. Lodish. 1983. pH and recycling of transferrin during receptor mediated endocytosis. Proc. Natl. Acad. Sci. USA. 80:2258-2262.

17. Jacobs, A. 1977. Low molecular weight intracellular iron transport compounds. Blood. 50:433-439.

18. Wright, S. D., and S. C. Silverstein. 1982. Tumor-promoting phorbol esters stimulate $\mathrm{C} 3 \mathrm{~b}$ and $\mathrm{C} 3 \mathrm{bi}$ receptor mediated phagocytosis in cultured human monocytes. J. Exp. Med. 156:1149-1164.

19. Kleber, E. E., J. D. Torrance, T. H. Bothwell, M. O. Simon, and R. W. Charlton. 1981. Mobilization of iron from peritoneal rat macrophages by desferrioxamine. Scand. J. Haematol. 27:209-218.

20. Lipschitz, D. A., J. Dugard, M. O. Simon, T. H. Bothwell, and R. W. Charlton. 1971. The site of action of desferrioxamine. Br.J. Haematol. 20:395-404.

21. Hershko, C., and T. E. A. Peto. 1988. Deferoxamine inhibition of malaria is independent of host iron status. J. Exp. Med. 168:375387.

22. Pollack, S., P. Aisen, F. D. Lasky, and G. Vanderhoff. 1976. Chelate mediated transfer of iron from transferrin to desferrioxamine. Br. J. Haematol. 34:231-235.

23. Bennett, J. P. 1978. Methods in binding studies. In Neurotransmitter Receptor Binding. H. I. Yamamura, S. J. Enna, and M. J. Kuhar, editors. Raven Press, New York. 71-74.

24. Horwitz, M. A. 1983. The Legionnaires' disease bacterium (Legionella pneumophila) inhibits phagosome-lysosome fusion in human monocytes. J. Exp. Med. 158:2108-2126.

25. Horwitz, M. A., and F. R. Maxfield. 1984. Legionella pneumo- 
phila inhibits acidification of its phagosome in human monocytes. $J$. Cell Biol. 99:1936-1943.

26. Stein, B. S., K. G. Bensch, and H. H. Sussman. 1984. Complete inhibition of transferrin recycling by monensin in K562 cells. J. Biol. Chem. 259:14762-14772.

27. Yamashiro, D. J., B. Tycko, S. R. Fluss, and F. R. Maxfield. 1984. Segregation of transferrin to a mildly acidic (pH 6.5) para-golgi compartment in the recycling pathway. Cell. 37:789-800.

28. Loo, V. G., and R. G. Lalonde. 1984. Role of iron in intracellular growth of Trypanosoma cruzi. Infect. Immun. 45:726-730.

29. Raventos-Suzrez, C., S. Pollack, and R. L. Nagel. 1982. Plasmodium falciparum: inhibition of in vitro growth by deferoxamine. Am. J. Trop. Med. Hyg. 31:919-922.

30. Griffith, E. 1987. Iron in biological systems. In Iron and Infection: Molecular, Physiological and Clinical Aspects. J. J. Bullen and E. Griffiths, editors. John Wiley \& Sons Ltd., Chichester, UK. 8-12.

31. Badwey, J. A., and M. L. Karnovsky. 1980. Active oxygen species and the functions of phagocytic leukocytes. Annu. Rev. Biochem. 49:695-726.

32. Locksley, R. M., R. F. Jacobs, C. B. Wilson, W. M. Weaver, and S. J. Klebanoff. 1982. Susceptibility of Legionella pneumophila to oxygen-dependent microbicidal systems. J. Immunol. 129:2192-2197.

33. Payne, N. R., and M. A. Horwitz. 1987. Phagocytosis of Legionella pneumophila is mediated by human complement receptors. $J$. Exp. Med. 166:1377-1389.

34. Yamamoto, K., and R. B. Johnston, Jr. 1984. Dissociation of phagocytosis from stimulation of the oxidative burst in macrophages. J. Exp. Med. 159:405-416.

35. Wright, S. D., and S. C. Silverstein. 1983. Receptors for C3b and $\mathrm{C} 3 \mathrm{bi}$ promote phagocytosis but not release of toxic oxygen from human phagocytes. J. Exp. Med. 158:2016-2023.

36. Aderem, A. A., S. D. Wright, S. C. Silverstein, and Z. A. Cohn. 1985. Ligated complement receptors do not activate the arachidonic acid cascade in resident peritoneal macrophages. J. Exp. Med. 161:617-622.

37. Jacobs, R. F., R. M. Locksley, C. B. Wilson, J. E. Haas, and S. J. Klebanoff. 1984. Interaction of primate alveolar macrophages and Legionella pneumophila. J. Clin. Invest. 73:1515-1523.

38. Friss, R. R. 1972. Interaction of L-cells and Chlamydia psittaci-entry of the parasite and host response to its development. $J$ Bacteriol. 110:706-721.

39. Jones, T. C., and J. G. Hirsch. 1972. The interaction between Toxoplasma gondii and mammalian cells. II. The absence of lysosomal fusion with phagocytic vacuoles containing living parasites. $J$. Exp. Med. 136:1173-1194.

40. Armstrong, J. A., and P. D'Arcy Hart. 1971. Response of cultured macrophages to Mycobacterium tuberculosis with observations on fusion of lysosomes with phagosomes. J. Exp. Med. 134:713-740

41. Alexander, J., and K. Vickerman. 1975. Fusion of host cell secondary lysosomes with the parasitophorous vacuoles of Leishmania mexicana-infected macrophages. J. Protozool. 22:502-508.

42. Chang, K. P., and D. M. Dwyer. 1976. Multiplication of a human parasite (Leishmania donovani) in phagolysosomes of hamster macrophages in vitro. Science (Wash. DC). 193:678-680.

43. Nogueira, N., and Z. A. Cohn. 1976. Trypanosoma cruzi. Mechanism of entry and intracellular fate in mammalian cells. J. Exp. Med. 143:1402-1420.

44. Tanowitz, H., M. Wittner, Y. Kress, and B. Bloom. 1975. Studies of in vitro infection by Trypanosoma cruzi. I. Ultrastructural studies on the invasion of macrophages and L-cells. Am. J. Trop. Med. Hyg. 25:25-33.

45. Nathan, C., N. Noguiera, C. Juangbhanich, J. Ellis, and Z. Cohn. 1979. Activation of macrophages in vivo and in vitro. Correlation between hydrogen peroxide release and killing of Trypanosoma cruzi. J. Exp. Med. 149:1056-1068.

46. Plefferkorn, E. R., M. Eckel, and S. Rebhum. 1986. Interferongamma suppresses the growth of Toxoplasma gondii in human fibroblasts through starvation for tryptophan. Mol. Biochem. Parasitol. 20:215-224.

47. Byrne, G. I., L. K. Lehmann, and G. J. Landry. 1986. Induction of tryptophan catabolism is the mechanism for gamma-interferon mediated inhibition of intracellular Chlamydia psittaci replication in T24 cells. Infect. Immun. 53:347-351.

48. Carlin, J. M., E. C. Borden, P. M. Sondel, and G. I. Byrne. 1987. Biologic response modifier-induced indoleamine 2,3 dioxygenase activity in human peripheral blood mononuclear cell cultures. $J$. Immunol. 139:2414-2418.

49. Nathan, C. F., T. J. Prendergast, M. E. Wiebe, E. R. Standley, E. Platzer, H. G. Remold, K. Welte, B. Y. Rubin, and H. W. Murray. 1984. Activation of human macrophages. Comparison of other cytokines with interferon. J. Exp. Med. 160:600-605.

50. Murray, H., A. Szuro-Sudol, D. Wellner, C. Rothermel, M. Oca, and A. Granger. 1988. Interferon induced tryptophan degradation and macrophage antimicrobial activity. Clin. Res. 36:580A.

51. Wilson, C. B. 1988. Molecular requirements for interferongamma induced macrophage anti-protozoal activity. Clin. Res. 36:582A. (Abstr.)

52. Hamilton, T. A., J. E. Weiel, and D. O. Adams. 1984. Expression of the transferrin receptor in murine peritoneal macrophages is modulated in the different stages of activation. J. Immunol. 132:2285-2290.

53. Hamilton, T. A., P. W. Gray, and D. O. Adams. 1984. Expression of the transferrin receptor on murine peritoneal macrophages is modulated by in vitro treatment with interferon-gamma. Cell. Immunol. 89:478-488. 